\title{
Simulation of Specular Surface Imaging Based on Computer Graphics: Application on a Vision Inspection System
}

\author{
Ralph Seulin \\ Laboratoire Le2i, CNRS FRE2309, EA 2421, Université de Bourgogne, 71200 Le Creusot, France \\ Email:r.seulin@iutlecreusot.u-bourgogne.fr \\ Frederic Merienne \\ Laboratoire Le2i, CNRS FRE2309, EA 2421, Université de Bourgogne, 71200 Le Creusot, France \\ Email: f.merienne@iutlecreusot.u-bourgogne.fr \\ Patrick Gorria \\ Laboratoire Le2i, CNRS FRE2309, EA 2421, Université de Bourgogne, 71200 Le Creusot, France \\ Email: p.gorria@iutlecreusot.u-bourgogne.fr
}

Received 31 July 2001 and in revised form 11 January 2002

\begin{abstract}
This work aims at detecting surface defects on reflecting industrial parts. A machine vision system, performing the detection of geometric aspect surface defects, is completely described. The revealing of defects is realized by a particular lighting device. It has been carefully designed to ensure the imaging of defects. The lighting system simplifies a lot the image processing for defect segmentation and so a real-time inspection of reflective products is possible. To bring help in the conception of imaging conditions, a complete simulation is proposed. The simulation, based on computer graphics, enables the rendering of realistic images. Simulation provides here a very efficient way to perform tests compared to the numerous attempts of manual experiments.
\end{abstract}

Keywords and phrases: reflective surface, online inspection, lighting, simulation.

\section{INTRODUCTION}

Highly reflective surfaces inspection is a problem met frequently within the automatic control of industrial parts $[1,2,3]$. This inspection is generally done manually. It implies subjectivity and tiredness influence on classification results. A machine vision system offers objectivity, better reliability and repeatability and is able to carry out defects measurement to classify the industrial parts quality.

This work aims at detecting surface defects on reflecting industrial parts. The objects to be controlled are highly reflective and so, act as perfect mirrors. Surface defects are dents, bumps, and scratches. The defects areas have the same reflective properties as the flawless area of the surface: they reflect incident light only in the specular direction. Industrial parts dimensions are $200 \times 50 \mathrm{~mm}$ and defects surface is less than $1 \mathrm{~mm}^{2}$.

This paper describes a complete machine vision system development. We will present the inspection system based on a particular lighting device that enables efficient real time defect detection and some of the features design performed via computer graphics simulation.

\section{SPECULAR SURFACE IMAGING AND DEFECTS DETECTION}

\subsection{Specular surface imaging}

\subsubsection{Lighting principle}

Imaging of reflective surfaces is not easy. We observe the entire object environment through its surface. In order to capture images without unwanted information, we need to completely master the environment of the surface. By choosing an adapted lighting system, the imaging of defects is possible. The lighting principle used in our system enables an efficient defects detection. A tried technique to reveal the aspect defects is the imaging of the reflection of a structured lighting through the surface $[1,2,3]$. The surface imperfections provoke important light rays' deviations. This property is used to detect defects with a particular lighting system. This lighting is binary type. It is composed of a succession of zones of null luminous intensity and zones of maximal luminous intensity. In these conditions, a defect appears in the captured image as a set of luminous pixels among a dark zone or a set 


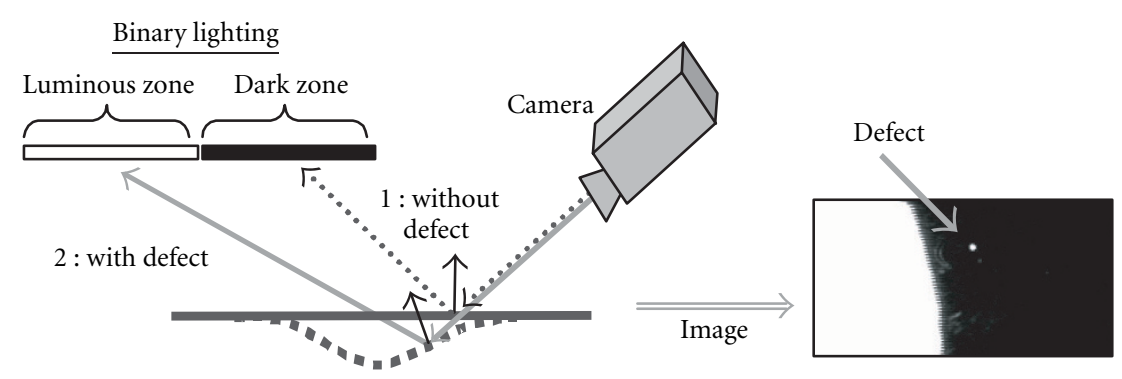

FIGURE 1: Lighting principle.

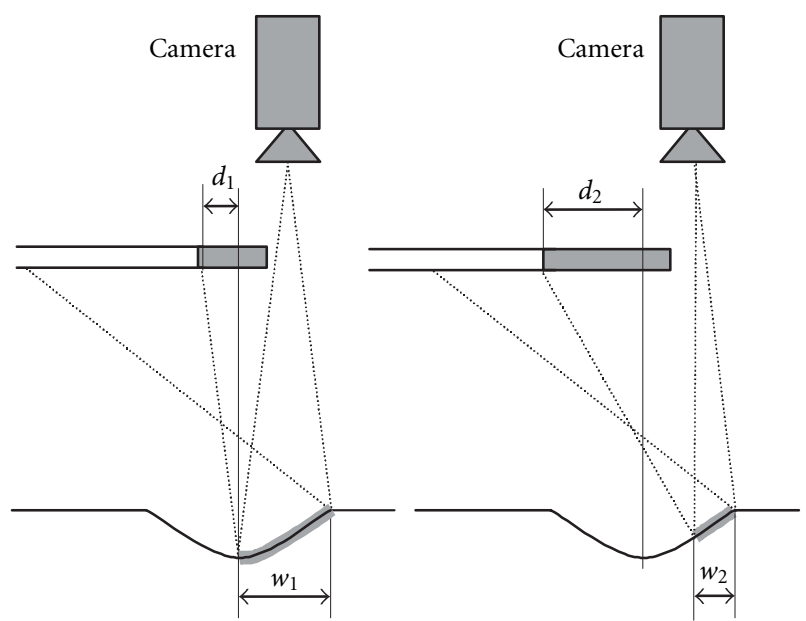

FIGURE 2: Defect size variation.

of dark pixels among a luminous zone. Figure 1 illustrates the lighting principle and shows a typical image acquired with the lighting device. In the first case, without defect, the surface reflects a dark zone of the lighting. In the second case, the defect deflects luminous rays coming from the luminous zone and so, the defect appears as a clear spot in a dark zone. We choose to saturate the camera in order to obtain images where defects appear very contrasted on a dark background and so to enable a simple image processing for detection (see Section 2.2). In these illumination and imaging conditions, defects appear only as high gray level pixels in dark zones.

\subsubsection{Implementation}

In order to inspect the whole part surface, an element of the lighting structure has to scan every part of the surface. During experiments, we noticed that the size of the defect signature on the image depends on the distance between the light transition and the defect. It can be schematically explained as shown in Figure 2.

If the light transition, projected on the surface, is close to the defect, the defect size on the image is close to its real size. But if the distance between the defect and the light transition increases, the defect size decreases and can even be null for an important distance. This particular property can be measured by computing the defect size from images acquired

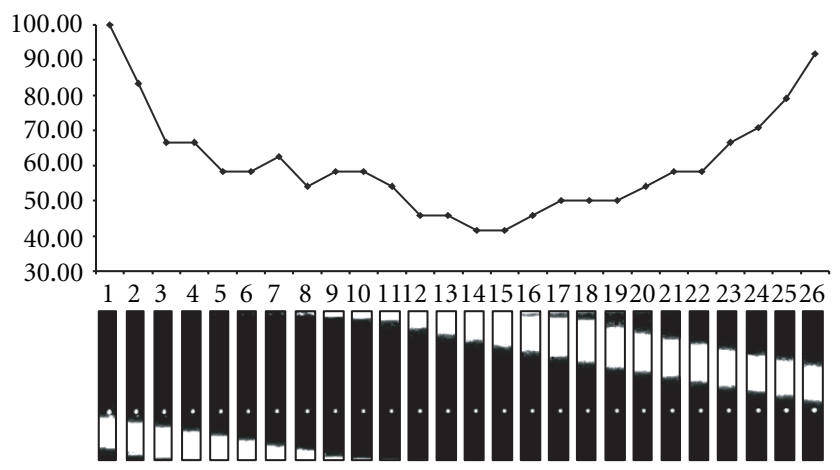

FIGURE 3: Defect size variation versus distance to the first light transition (experimental results).

during experiments. Figure 3 represents a defect size (percentage of real size) versus the distance between two light transitions and the center of the defect (normalized by the defect dimensions) and the corresponding images.

The image signature equals the physical size if the defect is close to a light transition and it decreases if the distance increases. In our industrial application, we have to obtain image signatures proportional to the defects physical size. So, the light transition has to scan all over the surface to ensure that each defect is close to a light transition in the image sequence. To carry out surface inspection, we can imagine that the object is moving in front of the camera and the lighting system [4]. In the case of important surface curvature gradients, the projection of the luminous and dark fringes on the complex geometry surface varies a lot between two consecutive images. So, entire scanning is not ensured if the object is moving in front of the static lighting. To overcome this limitation, an inverse process is proposed: the lighting structure is dynamic while the object is static.

Having static object during the inspection presents numerous advantages:

- the fringes projections and the position of the fringes between two images are completely mastered;

- an a priori knowledge of the object to be controlled can enable definition of region of interest in the surface inspection (see Section 2.2.2);

- shape defect detection can be computed by inspecting the silhouette. 
In order to reduce the number of necessary images to perform the scanning of industrial parts, the lighting system is composed of juxtaposed luminous and dark fringes. It enables a large number of light transitions to scan the surface. The lighting devices have to be diffuse and homogenous. So, the lighting system is realized by luminous surfaces made of diffusers placed in front of fluorescent tubes. The luminous panels are then shaded by an opaque mask.

The surface aspect imaging is performed by different lighting system positions. The lighting system is translated along the main object axis. For each regular spatial position, an image is captured. We finally obtain an image sequence as seen in Figure 4.

\subsection{Defects segmentation and measurements}

\subsubsection{Image sequence processing}

In the sequence, defects always appear as high gray level pixels because of the saturation of the Charge Couple Device (CCD) matrix. By computing the mean image of the sequence, we obtain a synthetic image called aspect image.In this image defects appear as high gray level pixels and the entire flawless area of the image appear with medium gray level (see Figure 5).

The segmentation of defects zones is then easy to compute because of the very contrasted aspect images. The segmentation processing consists in filtering the aspect image by a local $(9 \times 9$ pixels $)$ Gaussian filter and to subtract the resulting image to the initial one. With this filter, segmentation of pixels belonging to clear thin areas (defects) from zones of homogenous gray levels (flawless area) is performed.

\subsubsection{Postprocessing}

A postprocessing is then applied on the segmented image to distinguish holes or bumps from scratches and to compute defects measurements.

\section{Defects measurement}

Defects measurement consists first in blob coloring to label the defects. Then each defect is analyzed individually by computing moments on its representing pixels. Two-dimensional moments have been successfully used for a number of image processing applications $[5,6]$. For a digital image represented in a two-dimensional array, the moment of order $(p+q)$ is given by

$$
\boldsymbol{m}_{p q}=\sum_{x=0}^{M-1} \sum_{y=0}^{N-1} x^{p} y^{q} f(x, y),
$$

where $M$ and $N$ are the horizontal and vertical dimensions and $f(x, y)$ is the gray level at point $(x, y)$. The zeroth order moment, $\boldsymbol{m}_{00}$, represents the total mass of the given image. When computed for a silhouette image on a dark background, $\boldsymbol{m}_{00}$ represents the total object area. The two first order moments, $\boldsymbol{m}_{10}, \boldsymbol{m}_{01}$, are used to locate the center of mass of the object in the image. The coordinate of the center of mass $G\left(x_{G}, y_{G}\right)$ can be defined through moments as follows:

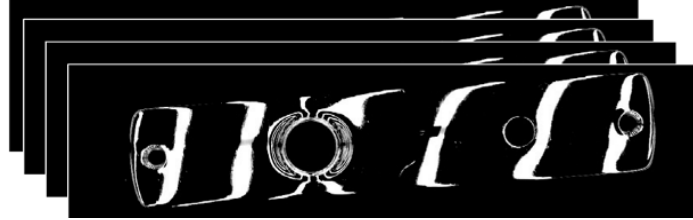

FIgURE 4: Part of image sequence (experimental results).

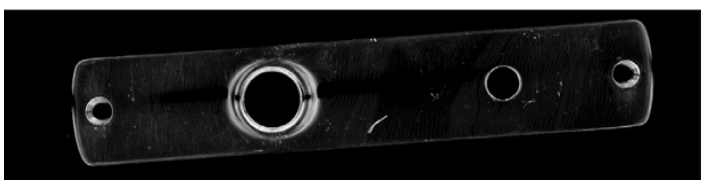

FIGURE 5: Aspect image (experimental results).

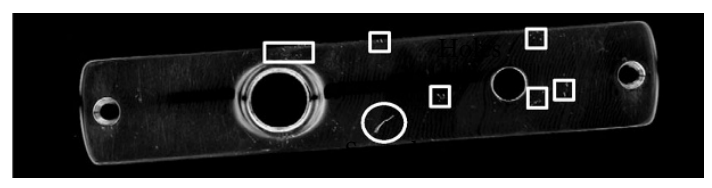

FIGURE 6: Segmented and classified defects.

$$
x_{G}=\frac{\boldsymbol{m}_{10}}{\boldsymbol{m}_{00}}, \quad y_{G}=\frac{\boldsymbol{m}_{01}}{\boldsymbol{m}_{00}} .
$$

So, by computing two-dimensional moments on the labeled image, the position (coordinate of the center of mass), the size (number of pixels representing the defect), and the weight (the sum of gray level pixels representing the defect in the aspect image) of each defect are computed. The defects are finally measured upon their size and weight.

The classification between bumps or holes versus scratches is made upon the compacity of defects (see Figure 6). The compacity of a shape is computed from the second-order central moments $\left\{\mu_{20}, \mu_{11}, \mu_{02}\right\}$, where

$$
\mu_{p q}=\sum_{x=0}^{M-1} \sum_{y=0}^{N-1}\left(x-x_{g}\right)^{p}\left(y-y_{g}\right)^{q} f(x, y)
$$

are the central moments of order $(p+q)$.

We consider that a shape is a hole or a bump if the following relation is checked:

$$
\frac{\mu_{02}-\mu_{20}}{\mu_{11}} \geq k \quad \text { or } \quad \frac{\mu_{11}}{\mu_{02}-\mu_{20}} \geq k,
$$

where $k$ is the chosen compacity factor. If the relation is not checked, the defect is considered as a scratch.

\section{Regions of interest}

The lighting principle used here is designed to reveal small geometrical surface imperfections. In the case of objects composed of smooth surfaces decorated by relief, the relief will be detected as pertaining to defects. So if we do not define regions of interest (ROI) for the defect detection, lots 


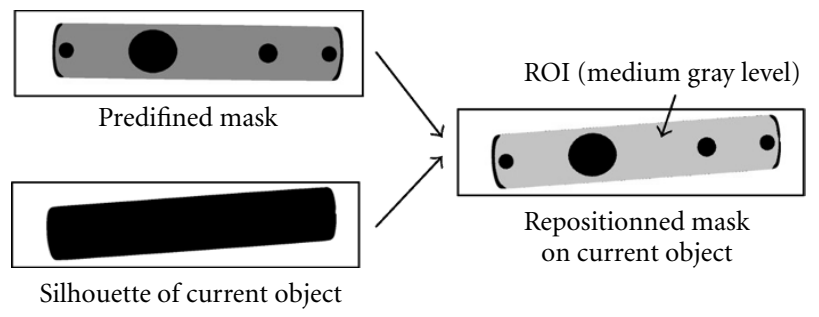

FIgure 7: Definition of ROI by equivalent ellipse matching.

of false detection will perturb the classification of parts. A method is so proposed to define ROI on the industrial parts. This method consists in positioning a predefined mask on the object to be controlled. The first phase is to compute the silhouette of the object from the image sequence. The silhouette is reconstructed from the image sequence. The image sequence represents the lighting scanning through the entire object surface. Then, by computing the sum of the $N$ images and by applying a flood fill method on the external reconstructed shape, the reconstruction of the object silhouette is effective. Once the silhouette obtained, we are able to match a predefined binary mask on the shape (see Figure 7). The matching is realized by fitting the equivalent ellipse of the shape to be controlled on the equivalent ellipse of the reference shape on which the binary mask is defined. The equivalent ellipse of a shape is an ellipse which has the same geometrical moments as the shape. In our case, the shape fitting is realized by matching the center of mass and the orientation of the two shapes. The center of mass is determined as described in Postprocessing (Section 2.2.2). The orientation $\alpha$ is computed from the three second-order central moments $\left\{\mu_{20}, \mu_{11}, \mu_{02}\right\}$ :

$$
\tan (2 \alpha)=\frac{2 \cdot \mu_{11}}{\mu_{02}-\mu_{20}} .
$$

The fitting is computed by scaling, translating, and rotating the reference shape to make it matching the current shape.

This method is applied in our case to perform the defect detection only in smooth surface areas. The main advantages of this method is the translation, rotation, and scale invariance. The position and orientation of the objects can be approximate. It simplifies consequently the parts manipulation and positioning on the production line.

\subsection{Summary of the method}

The specular surface inspection method can be summarized as shown in Figure 8.

\section{SPECULAR SURFACE IMAGING SIMULATION}

The imaging conditions have been particularly studied because they influence strongly the quality of acquired images and consequently, the quality of image processing results. These imaging conditions are often the fact of exper-

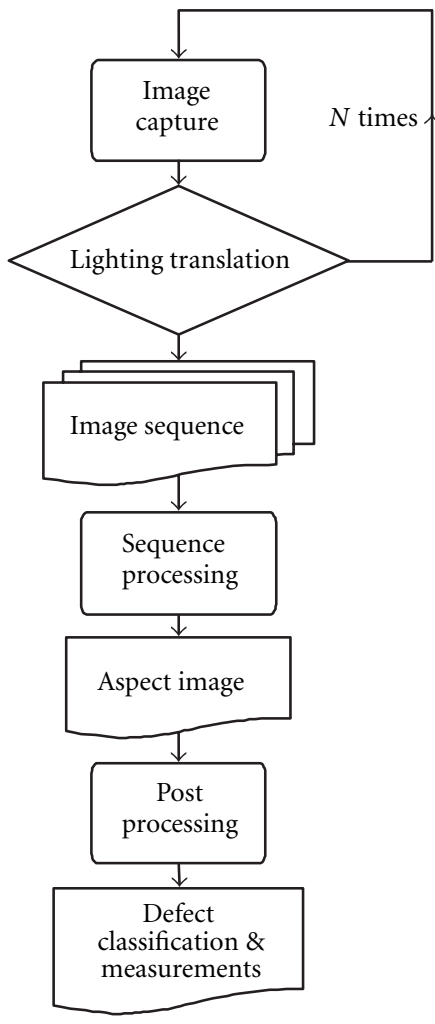

Figure 8: Specular surface inspection algorithm.

iments: numerous attempts on lighting features and on the relative positions between the camera, the lighting, and the object are still necessary. To bring help in the choice of these imaging features, a complete simulation of the system is proposed.

\subsection{Imaging system modeling}

Lighting and imaging features need to be modeled in order to simulate the imaging process. In our case, the geometrical optics is applicable because the wavelength of the incident light is weak compared to the dimensions of the surface imperfections [7]. The ray tracing technique is so used to analyze the reflection of the lighting through the surface. Each element of the scene is modeled and is described in this chapter.

\subsubsection{Camera model}

Because the geometrical optics is applicable, a pinhole model is used to describe the camera. The phenomenon of sensor saturation or blooming is extremely important within the framework of this application. It conditions the defect revealing, computed by the mean image of the sequence.

The model proposed here is based on experiments. It is applicable only in the case of high saturation imaging conditions. The camera saturation can be modeled by a blur effect and the resulting synthetic image is multiplied by a gain factor. Figure 9 presents an example of the saturation effect modeling. (a) is the experimental source image, (b) is the 


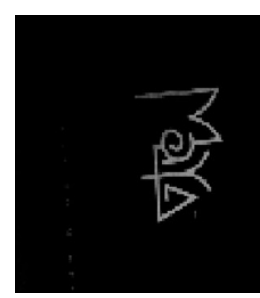

(a)

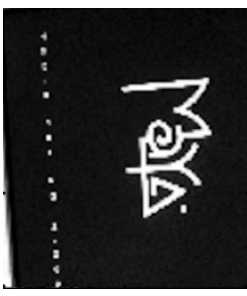

(b)

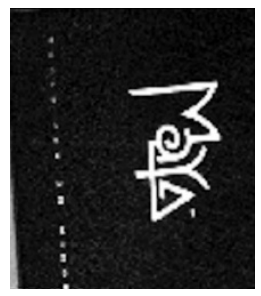

(c)

FIgURE 9: Saturation effect simulation.

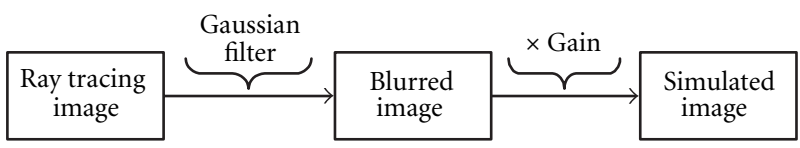

FIGURE 10: Saturation effect model.

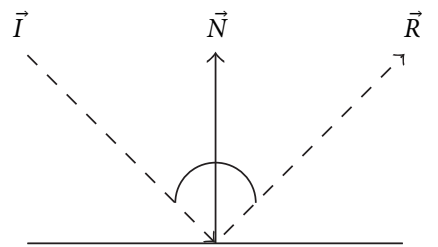

FIGURE 11: Lighting reflection properties on perfectly specular surfaces.

experimental saturated image, and (c) is the simulated saturated image from image (a).

To model the saturation effect, each image is processed by a filter (see Figure 10).

\subsubsection{Object model}

\section{Lighting reflection properties}

The reflection properties of the metal surfaces have been accurately studied [8]. A simple model is used here to describe the lighting reflection on the polished metal surface. We consider that the surface is perfectly specular. So, the Snell-Descartes law is applicable (see Figure 11). The light is reflected with the same angle as the incoming ray and the reflected luminous intensity is equal to the incoming one.

\section{Industrial parts model}

In the case of CAD/CAM (computer assisted design/ computer assisted manufacturing) designed and manufactured industrial parts, the CAD file is used for the object model. But most of times, the CAD model does not exist or is not sufficiently accurate for the modeling. In that case, we use a 3D scanner to obtain a range image of the surface to be controlled. The range image is then converted in a 3D model made of Non Uniform Rational B-Splines (NURBS) (see Figure 12).

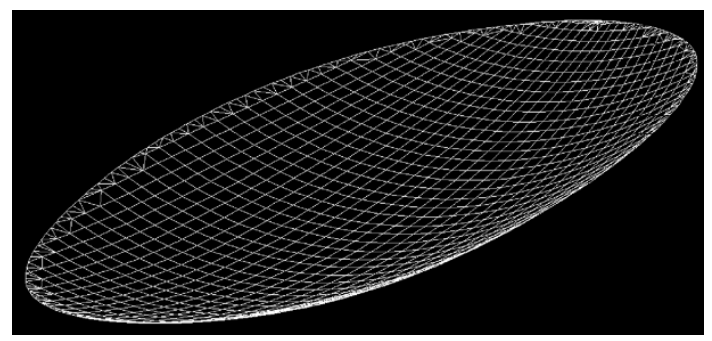

FIGURE 12: 3D object model.

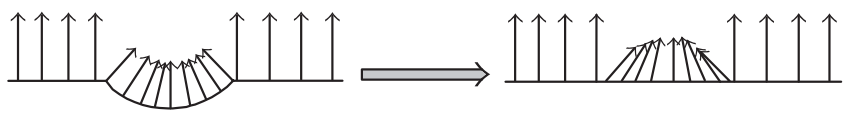

FIGURE 13: Bump mapping principle.

\section{Defects model}

Defects can be integrated in the 3D model of an object. Defects are zones where the surface is affected by geometrical imperfections. The surface is still smooth in the defect zones, so the specular property is maintained. For example, a dent is modeled by a Gaussian curve. The Gaussian function characterizes the smoothness of the surface.

The influence of surface height and surface orientation on the lighting point reflected by the surface has been studied [9]. This study is based on physical values taken from our machine vision prototype and is applicable in the case of a viewing point situated at a large distance from the object (compared to the defect dimensions). We demonstrate that the influence of the surface height induces negligible light rays' deviations compared to the influence of the surface orientation. The defect surface is so modeled as flat and is only described by its orientation. Normal vectors are computed from the surface model and completely describe the defect surface (see Figure 13). This kind of modeling can be compared to the bump mapping technique used in computer graphics to artificially represent the surface orientation modification $[10,11]$.

\subsubsection{Lighting model}

The lighting system was modeled in order to represent the developed prototype. The luminous zones are completely diffuse and homogenous. They can be modeled by Lambertian lighting sources. The lighting sources are shaded by opaque fringes where the luminous intensity is null. Figure 14 reminds the luminance law of a Lambertian lighting source. The luminous intensity varies with the observation angle $\theta$ of the lighting source with a cosine law.

\subsection{Simulation process}

The imaging and lighting model enables to simulate the image acquisition process. Ray tracing is used to compute realistic images. Ray tracing is well adapted for this kind of rendering because the surfaces are completely specular. Light rays 


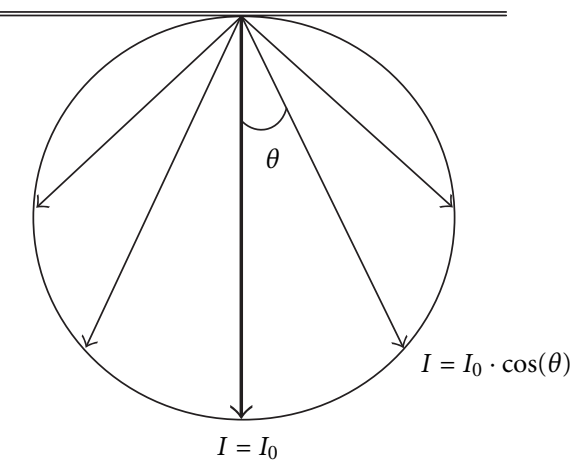

FIgURE 14: Lambertian lighting model.

are modeled by vectors. For each pixel $C$ of the CCD matrix, the lighting point $L$ reflected by the surface is computed from the reflected ray. Figure 15 describes this modeling.

Blue Moon Rendering Tools (BMRT) are used here for the rendering $[12,13]$. BMRT are a collection of programs that render 3D scene models. BMRT use some Application Program Interfaces (APIs) that are very similar to those described in the published RenderMan Interface Specification [14]. This rendering kernel has been chosen for its possible custom settings for all scene elements features. Each element of the scene is controlled by a shader. A shader describes the object handling on the light rays. There is so a shader for the lighting device which sets the Lambertian luminous fringes and the dark ones. And an object shader defines the surface as perfectly specular.

\section{APPLICATION}

\subsection{System features}

The method proposed in the first chapter is applied to the detection of surface defects on reflecting industrial parts. The system features have been first chosen by experimental tests. The tests have proved the feasibility of the detection and a first prototype has been developed.

The lighting stripes are disposed in a tunnel made of two flat panels. It enables so a complete mastery of the environment of industrial parts to be controlled. A high resolution CCD camera $(1300 \times 300$ pixels $)$, positioned vertically to the object plan, inspects the objects. We obtain a spatial resolution of $0.15 \mathrm{~mm}$ per pixel. Defects appear with an average size of 5 pixels on the images. Binary lighting zones dimensions and images sequence capture features are computed as described in [9]. The surface inspection is performed by several lighting system positions realized by a translation of the tunnel along the main object axis. A sequence of 24 images is captured during the lighting translation (see Figure 17). The camera captures images at a rate of 24 images per second, it enables to avoid the blur effect due to the translation movement. The acquisition conditions are schematically represented in Figure 16.

The homogeneity of the flawless area is critical in the defect segmentation. The imaging conditions have to be cho-

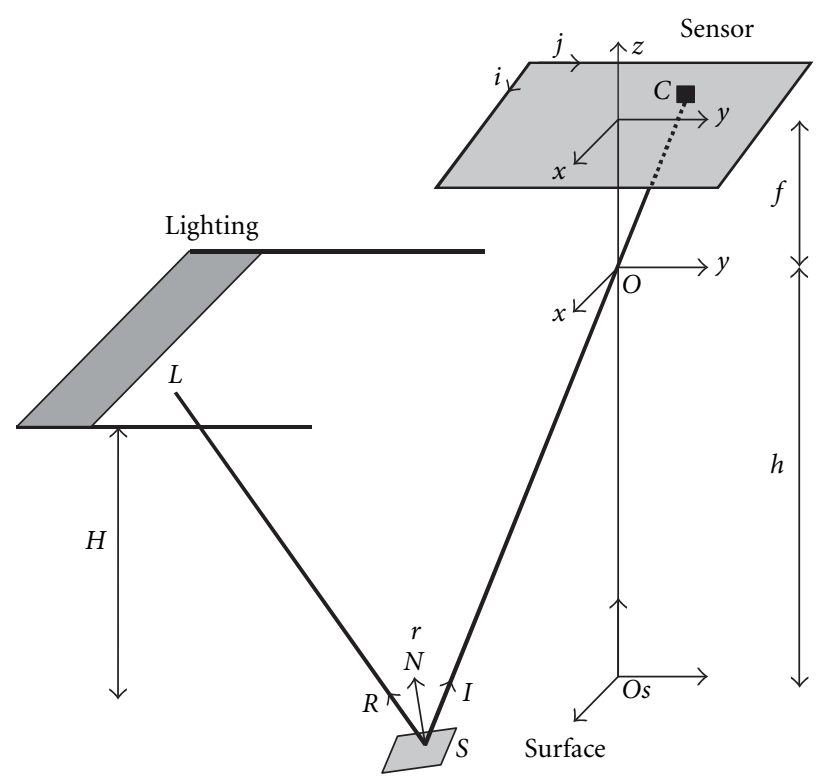

FIGURE 15: Imaging system modeling.

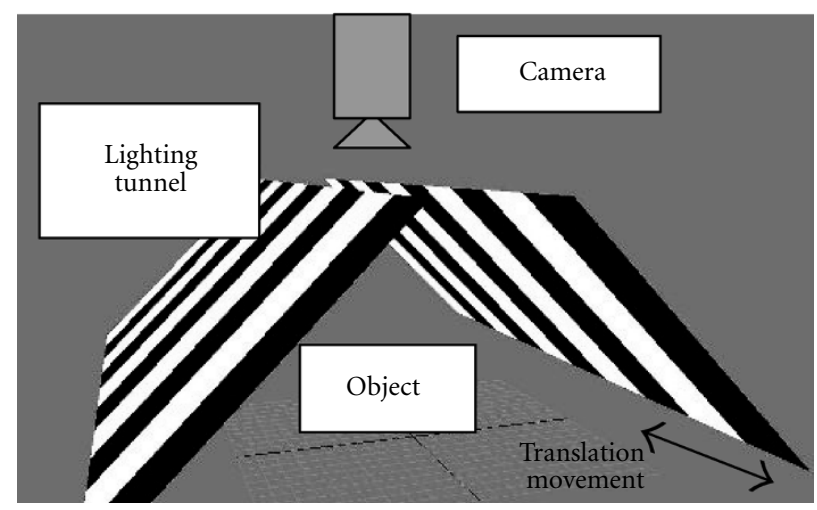

FIGURE 16: Specular surface imaging system (principle schema).

sen in order to provide aspect images with a flawless area the more homogenous as possible. The homogeneity criterion is estimated on aspect image by computing the following equation:

$$
h=1-\frac{\sum_{x=0}^{M-1} \sum_{y=0}^{N-1}|f(x, y) / \mu-1|}{M \times N}, \quad h \in[0,1],
$$

where $M$ and $N$ are the horizontal and vertical dimensions of the image, $f(x, y)$ is the gray level at point $(x, y)$, and $\mu$ is the mean luminance of the image, $h$ must be close to 1 or $100 \%$ to have a homogenous flawless area.

Figure 18 presents the aspect image resulting from the experimental sequence. The corresponding measured homogeneity is $h=85.6 \%$.

The images provided by the prototype can be obtained by simulation (see Figure 17). The simulation provides realistic images with the same features as the real ones. It completely 


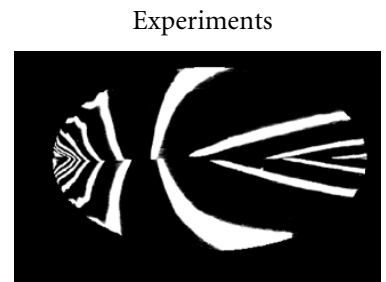

Lighting position 1

(a)

Experiments

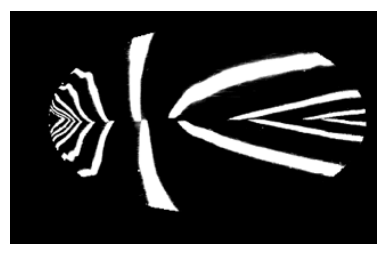

Lighting position 2

(c)

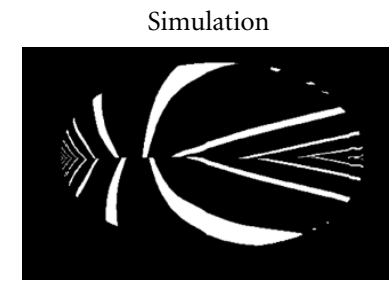

Lighting position 1

(b)

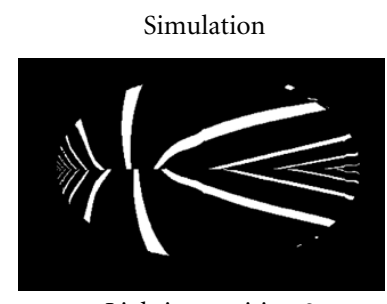

Lighting position 2

(d)
Figure 17: Part of image sequence (first experimental results and simulation).

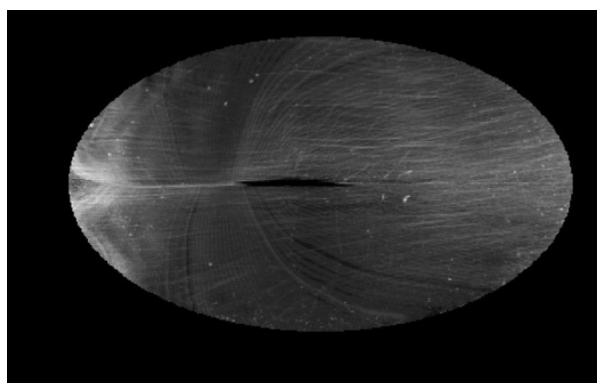

FIgURE 18: Aspect image form first experiments.

enables the simulation of industrial parts imaging. With the simulation, it is possible to simulate new imaging conditions and so to estimate the influence of scene elements features on the quality of acquired images and consequently the quality of image processing results. The simulation enables virtual tests on the acquisition conditions.

A new lighting system has been virtually tested by simulation. This new system uses a semicylindrical tunnel. Figure 19 presents a simulated image sequence with the new lighting configuration. It provides better images features than the first one: the regularity of the projected stripes is better and the resulting aspect images are more homogenous (see Figure 20). The corresponding measured homogeneity is $h=89.4 \%$.

Simulated tests have been so performed before construct-
Lighting position 1

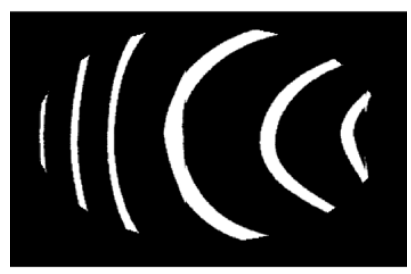

Lighting position 2

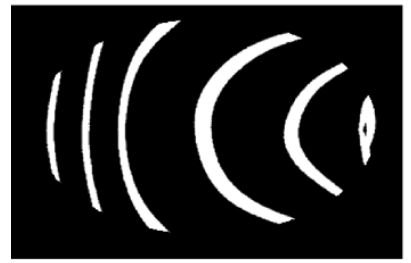

Lighting position 3

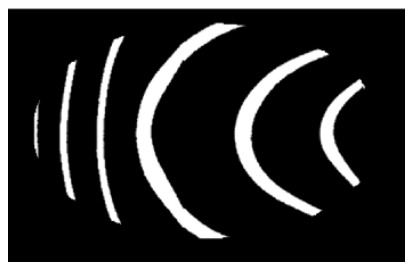

FIGURE 19: Part of image sequence (simulation results).

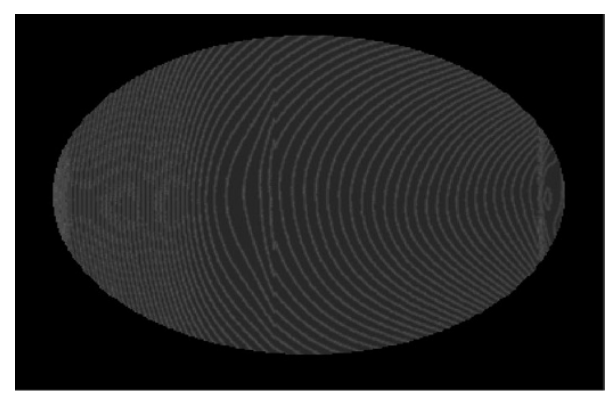

Figure 20: Aspect image resulting from the simulation.

ing a new and finalized prototype. Figure 21 presents a photograph of the final prototype and the real sequence provided by the new developed prototype is presented in Figure 22 . Figure 23 presents the aspect image resulting from the experimental sequence. The corresponding measured homogeneity is $h=88.8 \%$.

We can notice that the images features and homogeneity results provided by the prototype have been completely predicted by the simulation process. The simulation tool provides here a very efficient way to ease system improvement compared to the necessary numerous attempts of the manual experiments. This simulation tool brings help in the choice of the imaging and lighting parameters. 


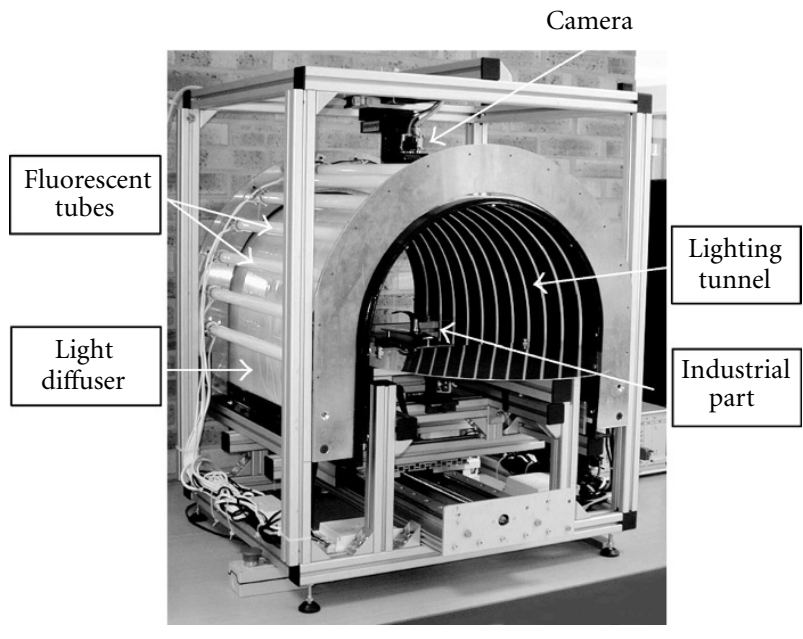

FIGURE 21: Developed machine vision system (prototype photograph).

\subsection{Performances}

The machine vision prototype has been tested on 200 industrial parts sorted out by an expert. The tests have been made on a selection of parts affected by all possible defects. This industrial parts selection is not representative of the production quality, but enables to estimate the classification possibilities of the machine vision system.

Two parameters were calculated. The first parameter is the size of the biggest defect detected on each part. The second parameter corresponds to the sum of the size of the detected defects. This last parameter is hence a function of the number of defects on the part. The graph of Figure 24 presents results obtained for holes defects. Each point corresponds to the quality measurement of a part. This point is placed on the graph according to the two parameters calculated. Three classes have been proposed by the expert (Perfect, Acceptable, and Rejected). For each parameter, a threshold has been defined to separate the three classes. The system is capable of distinguishing the class of defects proposed by the expert.

The diagram of Figure 25 presents results obtained for scratch defects. Two classes have been proposed by the expert (Perfect and Rejected). The parts are rejected as soon as they are not perfect. It is due to the segmentation processing for the scratches (see Section 2.2.2): if a scratch has a weak size, it is considered as a hole.

The machine vision prototype enables an efficient detection at the industrial production rate (1 part every 2 seconds). The sequence imaging time requires 1 second and the image processing takes 0.8 second (evaluated on a $700 \mathrm{MHz}$ Pentium III based machine).

\section{CONCLUSION AND FUTURE WORK}

A machine vision system for specular surface inspection has been presented. This system enables the detection of ge-
Lighting position 1

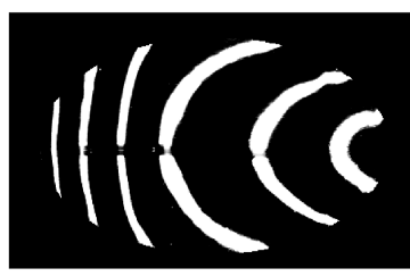

Lighting position 2

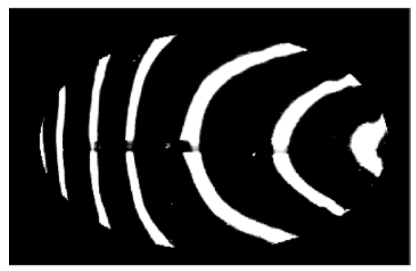

Lighting position 3

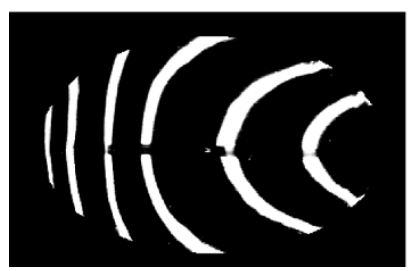

FIGURE 22: Part of image sequence (prototype results).

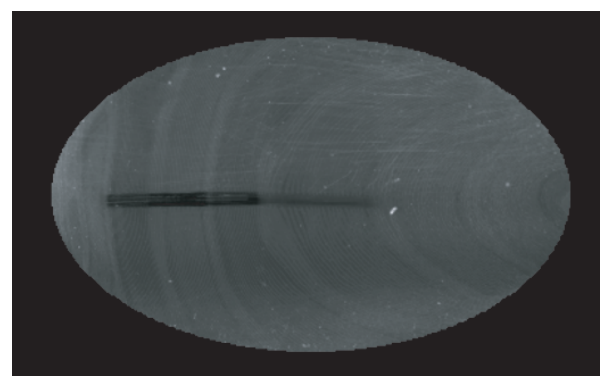

FIGURE 23: Aspect image provided by the prototype and measured homogeneity.

ometric aspect surface defects. The revealing of defects is realized by a particular lighting device. It has been carefully designed to ensure the imaging of defects. Defects appear very contrasted in resulting images. The elementary processing for defect segmentation, so very fast computing, is possible because of the well-designed lighting system. The lighting system simplifies a lot the image processing and so a real-time inspection of reflective products is thus possible. The imaging conditions have been particularly studied because they influence strongly acquired images. These imaging conditions are still often the fact of experiments. In order to bring help for the system conception, a complete simulation of the acquisition process have 


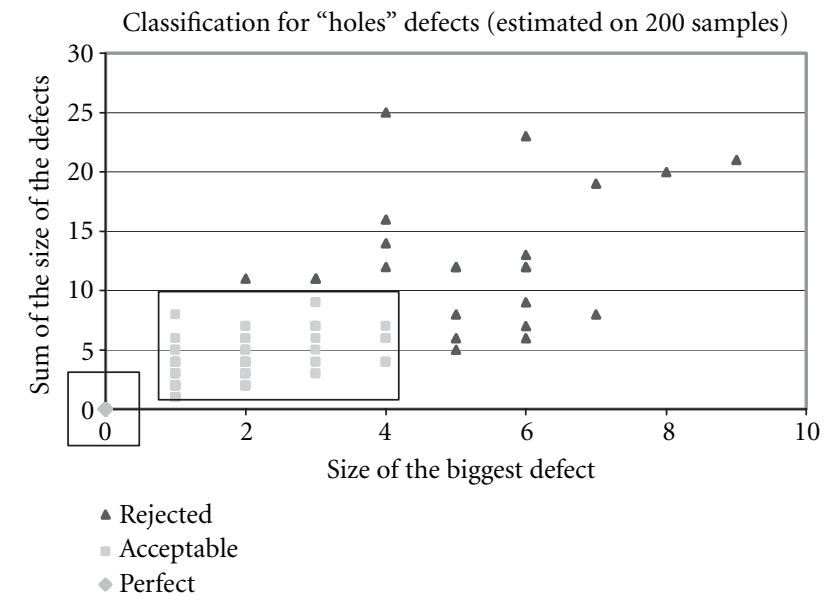

FIgURE 24: Classification of holes defects.

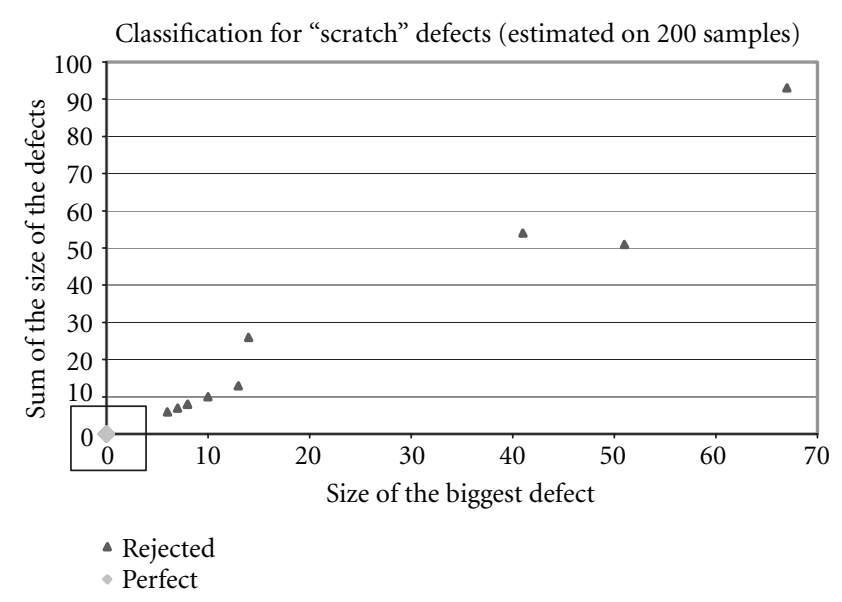

FIgure 25: Classification diagram for scratch defects.

been proposed. Each system device has been physically modeled and it enables to obtain realistic images. Simulation provides here a very efficient way to perform virtual tests compared to the necessary numerous attempts of the manual experiments. The use of simulation, based on computer graphics enables to save time in a machine vision system improvement.

Future work concerns the automatic optimization of the system via the simulation. The efficiency estimation of imaging conditions on the defect revealing and detection is studied. By measuring the quality of the resulting images, it will be possible to automatically optimize the system via a gradient-based method.

\section{ACKNOWLEDGMENT}

These research works have been realized with the financial support of the Regional Council of Burgundy.

\section{REFERENCES}

[1] C. Sanderson, L. E. Weiss, and S. K. Nayar, "Structured highlight inspection of specular surfaces," IEEE Trans. on Pattern Analysis and Machine Intelligence, vol. 10, no. 1, pp. 44-55, 1988.

[2] C. Bakolias and A. K. Forrest, "Dark field scheimflug imaging for surface inspection," in Proc. SPIE Machine Vision Applications in Industrial Inspection V, vol. 3029, pp. 57-68, San Jose, Calif, USA, February 1997.

[3] B. G. Batchelor, D. A. Hill, and D. C. Hodgson, Automated Visual Inspection-Chapter 7: Lighting and Viewing Techniques, IFS Publications, Bedford, England, and North Holland, Amsterdam, 1985.

[4] G. Delcroix, R. Seulin, B. Lamalle, P. Gorria, and F. Merienne, "Study of the imaging conditions and processing for the aspect control of specular surfaces," SPIE-Journal of Electronic Imaging, vol. 10, no. 1, pp. 196-202, 2001.

[5] Y. C. Chim, A. A. Kassim, and Y. Ibrahim, "Character recognition using statistical moments," in Image and Vision Computing, vol. 17, pp. 299-307, March 1999.

[6] M.-K. Hu, "Visual pattern recognition by moment invariants," IRE transactions on information theory, vol. 8, no. 2, pp. 179-187, 1962.

[7] S. K. Nayar, K. Ikeuchi, and T. Kanade, "Surface reflection: physical and geometrical perspectives," IEEE Trans. on Pattern Analysis and Machine Intelligence, vol. 13, no. 7, pp. 611-634, 1991.

[8] P. Kierkegaard, "Reflection properties of machined metal surfaces," Optical Engineering, vol. 35, no. 3, pp. 845-857, 1996.

[9] R. Seulin, F. Merienne, and P. Gorria, "Dynamic lighting system for specular surface inspection," in Machine Vision Applications in Industrial Inspection IX, vol. 4301 of SPIE Proceedings, San Jose, Calif, USA, 2001.

[10] J. F. Blinn, "Simulation of wrinkled surfaces," in Proc. Siggraph '78, Computer Graphics, vol. 12, pp. 286-292, 1978.

[11] J. J. Zhang, "Least distorted bump mapping onto surface patches," Computers and Graphics, vol. 22, no. 2-3, pp. 233242, 1998.

[12] L. Gritz and J. K. Hahn, "BMRT: a global illumination implementation of the RenderMan standard," Journal of Graphics Tools, vol. 1, no. 3, pp. 29-47, 1996.

[13] A. A. Apodaca and L. Gritz, "Advanced RenderMan: Creating CGI for motion pictures," in The Morgan Kaufmann Series in Computer Graphics and Geometric Modeling, December 1999, ISBN 1-55860-618-1. Official RenderMan website: http://www.pixar.com/.

[14] S. Upstill, The Renderman Companion: A Programer's Guide to Realistic Computer Graphics, Addison-Wesley, July 1989.

Ralph Seulin received a postgraduate diploma (DEA) in image processing from the University of Burgundy, France, in 1999. He is currently a Ph.D. student at the Le2i (Laboratoire d'Électronique, d'Informatique et d'Images) of the University of Burgundy, France. His research interests include lighting technology, industrial imaging modeling, simulation, specular surface inspection, and machine vision design.

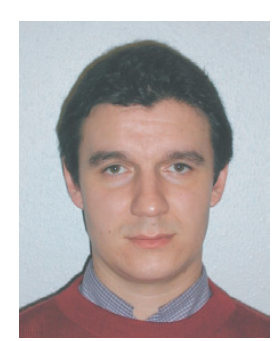


Frederic Merienne received his engineer's degree from the "École Nationale Supérieure d'Arts et Métiers" in 1992. He obtained his Ph.D. in January 1996 in electronics at the "Institut National Polytechnique de Grenoble." Since 1996, he is Maitre de Conferences at the Le2i, at the University of Burgundy, France. His research interests are focused on image processing for quality control by artificial

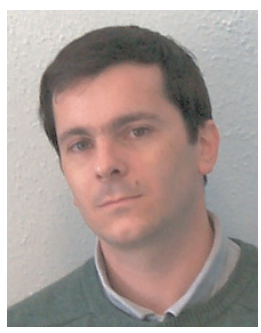
vision, the methods and tools for the simulation of imaging systems for the help design with computers graphics techniques, and the help design with virtual reality technologies.

Patrick Gorria received his engineering degree from the ENSAM, France, in 1980. He received his Ph.D. degree from the University of Burgundy, France, in 1984. Since 1993, he has been a professor at the Laboratory Le2i of Image Processing in the Institut Universitaire de Technologie (IUT) Le Creusot, where he is engaged in research and teaching on quality control by artificial vision. He has contributed to several areas

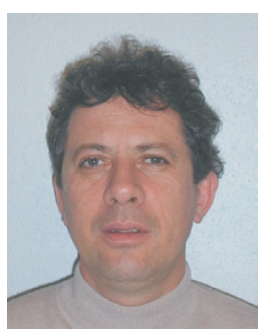
of classification, sensors, and other systems and applications using artificial vision systems for quality control. He is presently a principal researcher at Le2i, where he conducts research and development of defects detection for quality control by artificial vision; he holds several patents in the domain of image processing. 